\title{
Isolation, characterization and comparison of Atlantic and Chinook salmon growth hormone I and 2
} Kristian R von Schalburg1, Ryosuke Yazawa1, Johan de Boer1, Krzysztof P Lubieniecki², Benjamin Goh ${ }^{3}$, Christopher A Straub ${ }^{1}$, Marianne R Beetz-Sargent ${ }^{1}$, Adrienne Robb1, William S Davidson², Robert H Devlin ${ }^{3}$ and Ben F Koop*1

\author{
Address: ${ }^{1}$ Centre for Biomedical Research, University of Victoria, Victoria, British Columbia, V8W 3N5,Canada, ${ }^{2}$ Department of Molecular Biology \\ and Biochemistry, Simon Fraser University, Burnaby, British Columbia, V5A 1S6, Canada, and ${ }^{3}$ Fisheries and Oceans Canada, 4160 Marine Drive, \\ West Vancouver, British Columbia, V7V 1N6, Canada \\ Email: Kristian R von Schalburg - krvs@uvic.ca; Ryosuke Yazawa - ryo0520@yahoo.co.jp; Johan de Boer - jdboer@uvic.ca; \\ Krzysztof P Lubieniecki - klubien@sfu.ca; Benjamin Goh - GohB@pac.dfo-mpo.gc.ca; Christopher A Straub - cstraub@uvic.ca; \\ Marianne R Beetz-Sargent - marianbs@uvic.ca; Adrienne Robb - arobb@uvic.ca; William S Davidson - wdavidso@sfu.ca; \\ Robert H Devlin - DevlinR@pac.dfo-mpo.gc.ca; Ben F Koop* - bkoop@uvic.ca \\ * Corresponding author
}

Published: 3 November 2008

BMC Genomics 2008, 9:522 doi:10.1186/1471-2164-9-522
Received: 9 May 2008

Accepted: 3 November 2008

This article is available from: http://www.biomedcentral.com//47I-2/64/9/522

(c) 2008 von Schalburg et al; licensee BioMed Central Ltd.

This is an Open Access article distributed under the terms of the Creative Commons Attribution License (http://creativecommons.org/licenses/by/2.0), which permits unrestricted use, distribution, and reproduction in any medium, provided the original work is properly cited.

\begin{abstract}
Background: Growth hormone (GH) is an important regulator of skeletal growth, as well as other adapted processes in salmonids. The GH gene $(g h)$ in salmonids is represented by duplicated, non-allelic isoforms designated as ghl and gh2. We have isolated and characterized gh-containing bacterial artificial chromosomes (BACs) of both Atlantic and Chinook salmon (Salmo salar and Oncorhynchus tshawytscha) in order to further elucidate our understanding of the conservation and regulation of these loci.

Results: BACs containing ghl and gh2 from both Atlantic and Chinook salmon were assembled, annotated, and compared to each other in their coding, intronic, regulatory, and flanking regions. These BACs also contain the genes for skeletal muscle sodium channel oriented in the same direction. The sequences of the genes for interferon alpha-I, myosin alkali light chain and microtubule associated protein Tau were also identified, and found in opposite orientations relative to $\mathrm{ghl}$ and gh2. Viability of each of these genes was examined by PCR. We show that transposon insertions have occurred differently in the promoters of gh, within and between each species. Other differences within the promoters and intronic and 3'-flanking regions of the four gh genes provide evidence that they have distinct regulatory modes and possibly act to function differently and/or during different times of salmonid development.

Conclusion: A core proximal promoter for transcription of both $\mathrm{gh} /$ and gh2 is conserved between the two species of salmon. Nevertheless, transposon integration and regulatory element differences do exist between the promoters of $g h l$ and gh2. Additionally, organization of transposon families into the BACs containing ghl and for the BACs containing gh2, are very similar within orthologous regions, but much less clear conservation is apparent in comparisons between the $\mathrm{ghl}$ - and gh2-containing paralogous BACs for the two fish species. This is consistent with the hypothesis that a burst of transposition activity occurred during the speciation events which led to Atlantic and Pacific salmon. The Chinook and other Oncorhynchus GHIs are strikingly different in comparison to the other $\mathrm{GHs}$ and this change is not apparent in the surrounding non-coding sequences.
\end{abstract}




\section{Background}

Salmonids are used as models for studies in environmental toxicology, physiology, comparative immunology, growth, gametogenesis, olfaction and osmoregulation [1]. Although considerable knowledge of the basic biology of salmonids exists [1-3], the characterization of salmonid genomes will better enable scientific decisions on the conservation and enhancement of wild stocks, improve knowledge of fish health and increase the commercial viability of aquaculture. Sequencing of salmonid genomes also permits investigators to study fundamental questions concerning genome evolution, genome duplication and rearrangements, repeat-rich structures, transposon activity, gene silencing, and the re-establishment of a more stable diploid genome from a pseudotetraploid state.

Growth hormone $(\mathrm{GH})$ plays a very important role in many regulatory, metabolic and developmental processes in various vertebrate tissues [4]. In salmonids, GH is the principle stimulator of skeletal growth and plays a key role in lipid mobilization, protein synthesis and feeding behaviour [5]. In various fishes, GH also manifests some functions not found in all vertebrates, such as activities that influence sexual maturation and saltwater adaptation $[6,7]$. The GH gene $(g h)$ in salmonids is represented by duplicated, non-allelic isoforms designated as gh1 and gh2 [8], which diverged at least 30 million years (MY) ago [9].

In vertebrates, the major source of $\mathrm{GH}$ is the somatotrophic cells in the pituitary, from which it is secreted into and borne by the plasma to act on receptors throughout the organism $[5,10]$. Splice variants have also been isolated from extrapituitary tissue in humans (placenta, testis, blood mononuclear cells) and chicken (eye, heart) [[11] and refs. therein]. In trout, $g h$ transcripts have been detected in the pituitary, liver, head kidney, spleen, thymus, intestine and leukocytes and gut $[12,13]$. Improved understanding of the chromosomal environment in which $g h$ genes reside in salmonids will assist our understanding of the transcriptional controls regulating these genes. By analyzing two paralogous genes (e.g. gh1 and gh2) and doing so in two species (which have been separated for approximately $20 \mathrm{MY}$; [9]), identification of common conserved regions with presumed broad functional importance can be achieved. Further, regions which are found to be conserved only between species in one paralogue type may be important for differential regulatory control between the paralogues, while differences between species within a single paralogue type may help identify regions not important to regulation. Regions which are conserved between paralogues in only one species are candidates for regions which have undergone gene conversion subsequent to divergence between the two species. While gene conversion does not appear to have occurred at salmonid GH loci when examined at the gene level [9], an analysis of gene conversion has not been examined at larger scales now possible with genomic analyses. Understanding these sequence relationships has important practical ramifications since complete genomic resources are being developed for Atlantic salmon. It is important to determine the degree of conservation of this information with other important salmonid species such as Chinook salmon for extrapolation of emerging genomic information from one species to another.

Bacterial artificial chromosomes (BACs) containing gh1 and gh2 from both Atlantic and Chinook salmon were assembled, annotated, and compared to each other in their coding, intronic, regulatory, and flanking regions. A core proximal promoter for transcription of both $g h 1$ and gh2 is conserved between the two species of salmon. A $1600 \mathrm{bp}$ insertion of a Tc1-like DNA transposon sequence was found within the promoter region of both gh2 genes, but not in the promoter of the ghl genes. Furthermore, a Polinton-1 transposon is inserted in only the promoter for Chinook salmon GH1. Other differences within the promoters and intronic and 3'-flanking regions of the four genes provide evidence that supports the notion that they are regulated differently and thus may possess different functions. Intriguingly, Chinook salmon GH1 has undergone more than twice as many changes than any of the other GHs; changes not reflected in the surrounding noncoding DNA.

\section{Results}

Confirmation that the Atlantic salmon BACs we isolated were gh-containing BACs was performed by comparing HindIII-digested BAC fragment profiles to profiles on the internet Contig Explorer version 3.4 (iCE 3.4) database [14]. The gene for the skeletal muscle sodium channel (scn) was also identified by PCR for each isolated BAC, suggesting that each BAC contained the 5'-region upstream of each $g h$ gene. $g h$ type ( $g h 1$ vs. $g h 2$ ) was determined for BACs in both Atlantic and Chinook salmon by paralogue-specific PCR.

\section{BAC and $\mathrm{GH}$ comparisons}

Atlantic salmon (AS) and Chinook salmon (CS)gh loci were analyzed using DIGIT [15], which identified the presence, location, and direction of putative genes on each BAC. Each of these putative genes were assessed by BLASTX [16] to protein databases. BACs containing the $g h$ genes also bore the genes for scn oriented in the same direction (Figure 1A). The sequences of the genes for interferon alpha-1 (ifna1), myosin alkali light chain ( $\mathrm{mlc}$ ) and microtubule associated protein Tau (mapt) were also identified, and found in opposite orientations relative to gh1 and gh2 (Figure 1A). The Chinook salmon gh1 and gh2containing BACs are similarly organized. 

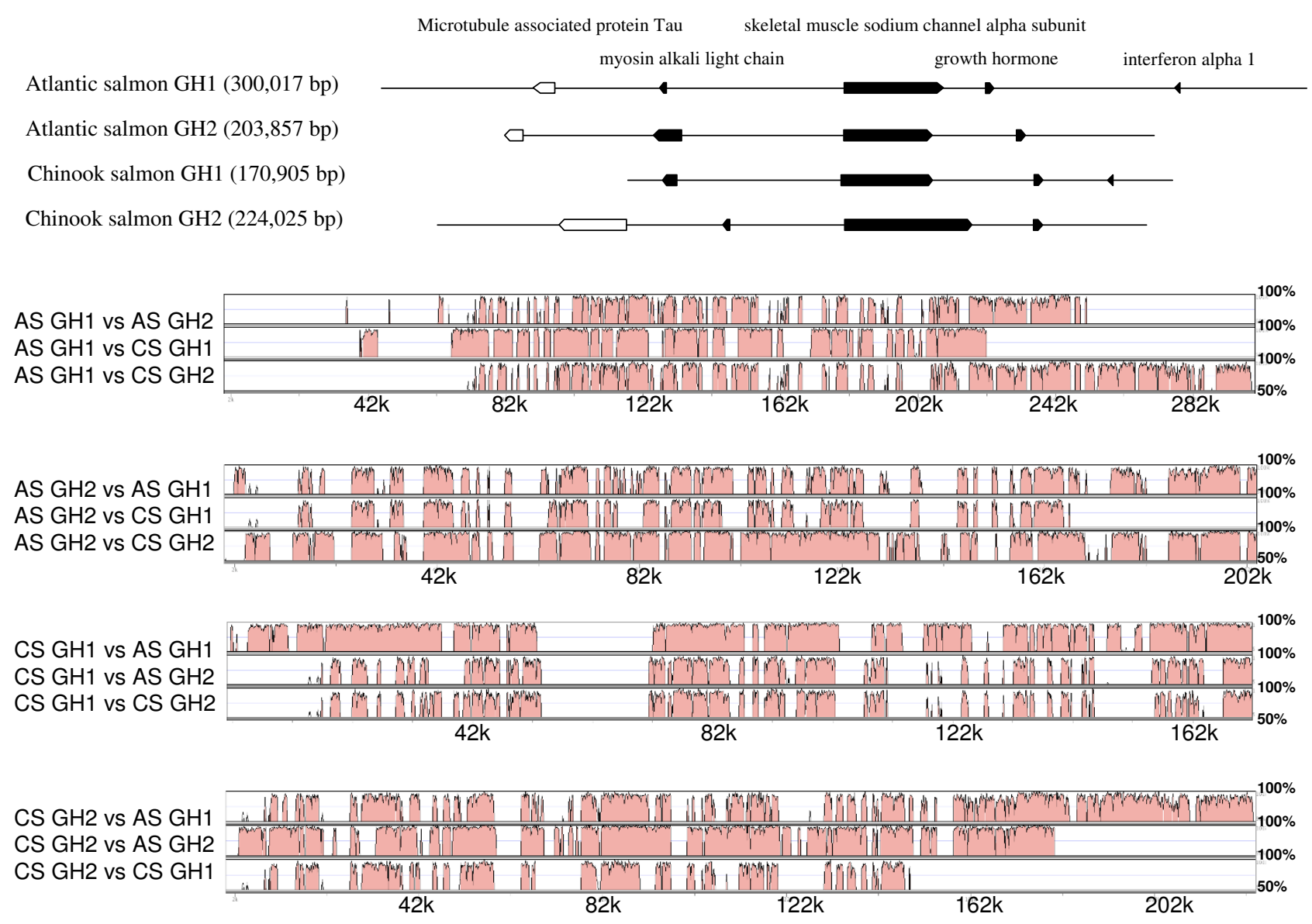

\section{Figure I}

A) The genomic organization of growth hormone I and 2 gene loci in Atlantic and Chinook salmon. Approximate sizes of the sequenced bacterial artificial chromosomes are indicated in parentheses. Growth hormone $\mathrm{I}$ and 2 genes and their flanking genes are represented by boxes with transcriptional directions indicated by arrowheads. The introns for the genes are not shown. Pseudogenes are indicated by open boxes. B) A comparison of Atlantic salmon and Chinook salmon gh I and gh2 loci shown in VISTA plots. Regions of high similarity (50 to I00\%; vertical axis) are separated by gaps that represent repeat sequences, deletions and insertions. Orthologous comparisons (AS ghl and CS ghl, and AS gh2 and CS gh2) appear more similar than paralogous comparisons (ghl and gh2).

An overall comparison of Atlantic and Chinook salmon gh1 and gh2 loci are shown in VISTA plots [17] (Figure $1 \mathrm{~B})$. This figure shows many regions of high similarity separated by gaps that represent repeat sequences, deletions and insertions. Visually orthologous comparisons (AS gh1 and CS gh1, and AS gh2 and CS gh2) appear more similar than paralogous comparisons ( $g h 1$ and $g h 2)$.

Using RepeatMasker [18] and a new salmonid repeat database [19], 30\% of the CS gh1, 28\% of the CS $g h 2,33 \%$ of AS gh 1 and $35 \%$ of AS gh2 are repeated elements or simple repeats (approximately 50\% of the total repeats are $1.6 \mathrm{~kb}$ Tc1-like transposons). With repeats removed from the genomic sequences, paralogous comparisons showed that Atlantic salmon $g h 1$ and gh2 have $89.5 \%$ identity over $46,425 \mathrm{bp}$, and Chinook salmon gh1 and gh2 showed $87.9 \%$ identity over 52,782 bp. These alignments also revealed numerous insertions and deletions among these loci.

In comparisons of Atlantic and Chinook salmon BACs, gh1 showed $92.3 \%$ identity over 85,987 bp and $g h 2$ showed $93.4 \%$ identity over 78,106 bp. Estimates of 93\% identity between Atlantic salmon and Chinook salmon are similar to the $94 \%$ identity found between Atlantic 
salmon and rainbow trout (Oncorhynchus mykiss) over 125 $\mathrm{kb}[20]$.

Comparisons of the 210 amino acid residues of GH show very strong similarity (Figure 2). Atlantic salmon GH1 and GH2 shows 97.1\% identity, while Chinook salmon GH1 and GH2 shows 93.1\% identity, and Atlantic salmon and Chinook salmon GH1 and GH2 shows 94.1\% identity and $97.1 \%$ identity, respectively. An analysis of these relationships indicates that the CS GH1 has undergone more than twice as many changes than for any of the other GHs. This change in CS GH1 is not apparent in the surrounding non-coding sequences and indicates there may have been a shift of natural selection pressures on the coding region of the CS gh1.

\section{Gene annotation}

The gh paralogs were fully annotated and the genes consist of 6 exons and 5 introns. All upstream positions we document are presented in relationship to the translation start site (in negative numbers) and, due to variations in insertions and deletions between the gh genes within their intronic regions, all downstream information is based on nucleotide (nt) numbering as presented in Additional file 1 .

\section{GH promoter and intron analysis}

A TATA box for all four $g h$ genes is found at position -94 to -87 [see Additional file 1]. Each gene has an asymmetric cAMP-response element (CRE) (TGACG) at -217/-213 in the proximal promoter and a palindromic CRE at position $3563 / 3570$ within intron 4 , but the gh2 genes have single nt substitutions in the fourth base at this location [see Additional file 1]. There are at least four potential pituitary transcriptional activator-1 (Pit-1) binding elements (coordinates: $-351 /-338,-249 /-237,-147 /-134$, and -119/ -106) that are shared by each gene and two upstream, reverse-oriented Pit- 1 binding motifs in only the gh2 promoters (TATGTTATTTAAAC). A retinoic acid receptor/ retinoid $\times$ receptor $(\mathrm{RAR} / \mathrm{RXR})$ binding element is also located here at position -190 to -171 . A potential estrogen response element (ERE) can be found in each promoter (GGCCAnnnTGACA), roughly 6,600 bp and 12,000 bp upstream of the transcription start site in both the $g h 1$ and gh2 genes, respectively. Another potential ERE (GGTCAnnnnnTGAGC), found in only the gh2 promoters, is located about 6,200 bp and 10,200 bp upstream of the CS and AS gh genes, respectively.

Intron 3 of the gh 2 genes are much shorter than the corresponding intron for the gh1 paralogs, partly accounted for by insertion of SSsp2201 microsatellites within them. Intron 5 of CS $g h 1$ is at least 360 nts longer than for any of the other genes. Also, numerous polymorphic loci exist

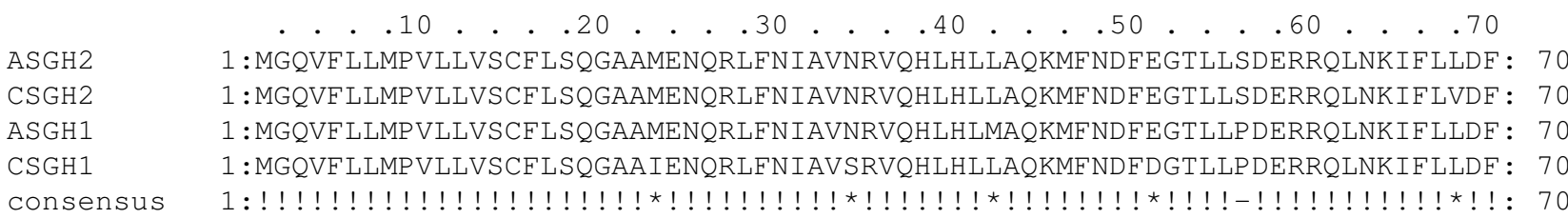

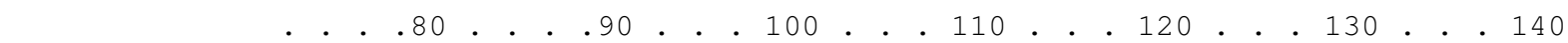

ASGH2 71 :CNSDSIVSPIDKQETQKSSVLKLLHISFRLIESWEYPSQTLAISNSLMVRNSNQISEKLSDLKVGINLLI: 140

CSGH2 $71:$ CNSDSIVSPIDKQETQKSSVLKLLHISFRLIESWEYPSQTLTISNSLMVRNYNQISEKLSDLKVGINLLI: 140

ASGH1 71:CNSDSIVSPIDKLETQKSSVLKLLHISFRLIESWEYPSQTLTISNSLMVRNSNQISEKLSDLKVGINLLI:140

CSGH1 71 : CNSDSIVSPVDKHETQKSSVLKLLHISFRLIESWEYPSQTLIISNSLMVRNANQISEKLSDLKVGINLLI : 140

consensus 71 : ! ! ! ! ! ! ! !* ! ! - ! ! ! ! ! ! ! ! ! ! ! ! ! ! ! ! ! ! ! ! ! ! ! ! ! ! ! ! ! ! - ! ! ! ! ! ! ! ! ! ! ! ! ! ! ! 140

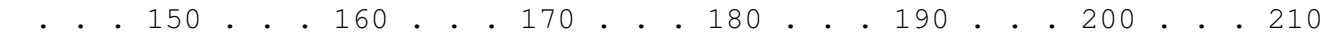

ASGH2 $141:$ KGSQDGVLSLDDNDSQHLPPYGNYYQNLGGDGNIRRNYELLACFKKDMHKVETYLTVAKCRKSLEANCTL:210

CSGH2 141:KGSQDGVLSLDDNDSLHLPPYGNYYQNLGGDGNVRRNYELLACFKKDMHKVETYLTVAKCRKSLEANCTL:210

ASGH1 $141:$ KGSQDGVLSLDDNDSQQLPPYGNYYQNLGGDGNVRRNYELLACFKKDMHKVETYLTVAKCRKSLEANCTL:210

CSGH1 141:TGSQDGLLSLDDNDSQQLPPYGNYYQNLGGDGNVRRNYELLACFKKDMHKVETYLTVAKCRKSLEANCTL:210

consensus 141 : $^{\star}$ ! ! ! !* ! ! ! ! ! ! !*- ! ! ! ! ! ! ! ! ! ! ! ! ! !* ! ! ! ! ! ! ! ! ! ! ! ! ! ! ! ! ! ! ! ! ! ! ! ! ! ! ! ! ! ! ! 210

\section{Figure 2}

Amino acid residue alignments of Atlantic and Chinook salmon $\mathbf{G H I}$ and 2. Identical residues designated by exclamation marks; asterisks denote strong residue similarity. 
in intron 4 of the gh genes that have been previously characterized for salmonids, including AS and CS (coordinates: $2895 / 2917 ; 3060 / 3083 ; 3635 / 3673$ and $3822 /$ 3865) [8]. The sequences from position 3060 to 3083 match identically [see Additional file 1], but we show that there is one nucleotide difference in the AS gh1 paralogue at position 3069. All of the other polymorphic loci match identically.

There are three polyadenylation (poly(A)) tail signal sequences (AATAAA) beginning at positions 5264, 5617 and 5717 [see Additional file 1]. All four $g h$ genes have at least one poly(A) signal sequence, but only the gh1 genes potentially possess three canonical signal sequences.

\section{Transposon integration analysis}

A Tc1-like DNA transposon sequence (Tss, [Genbank:L12207]) is inserted into the promoter region of both AS and CS gh2, approximately 2000 bp upstream of the transcription initiation site (1897 bp in AS; 2057 bp in CS) (Figure 3). This transposon is not found in the promoters of $g h 1$ for either species. This places the insertion of this transposon between the time of the gene duplication and when the two species diverged. A second Tc1-like transposon, DTSsa2 [Genbank:EF685955], is inserted approximately $7 \mathrm{~kb}$ upstream of the initiation site in only the AS gh2 promoter. In addition, a $1.2 \mathrm{~kb}$ fragment of SsaRT.3, a non-LTR long interspersed nuclear element (LINE) sequence identified in AS (unpublished data), is inserted approximately $1420 \mathrm{bp} 5^{\prime}$ of the transcription start site in CS gh1 (Figure 3). This fragment is truncated at the 3'-end, consistent with partial retrotranscription. Furthermore, an unusual insertion $10 \mathrm{~kb}$ upstream of only the CS gh1 gene was found (Figure 3). This insertion is approximately $20 \mathrm{~kb}$ in length containing a $10 \mathrm{~kb}$ palindromic sequence which is part of a Polinton- 1 transposon (Figure 4) [21].

\section{Tissue expression PCRs}

To examine the functionality of the genes identified on each gh-containing BAC, primers specific for each gene were designed and used in PCRs on a panel of tissues for each species [see Additional files 2 and 3]. This was not done for genes that were clearly pseudogenic or for genes for which information was incomplete, such as the genes that encode dynein heavy chain (data not shown) and microtubule associated protein Tau. Microtubule associated protein Tau is pseudogenic due to frameshifts and stop codons within the gene in each species (data not shown).

We conducted tissue expression studies of the 7 genes we had characterized in the BACs to determine if similarities existed in expression pattern between the two species; if expression was confined to specific tissues and whether indeed each of these genes were functional. Of the 7 genes examined, the expression of the gh paralogues was the least diverse. The expression of $g h 1$ and $g h 2$ was restricted to only muscle, brain, gill or eye (pituitary gland was not examined). Expression of the scn genes was not restricted to just skeletal muscle, but indeed was found in every tissue except the skin. It is also evident that ifna1 has fairly broad expression in the tissues examined here.

Myosin alkali light chain 1 also was expressed in a large range of different tissues. However, myosin alkali light chain $2(\mathrm{mlc} 2)$ appears to not be expressed at detectable levels in any of the tissues we examined in either species, despite in silico analysis indicating the viability of the $m l c 2$ transcript. Each gene that encodes $m l c 2$ appears intact, with no obvious problems for transcription or translation.

\section{Discussion \\ GH transcription}

Transactivation and footprinting studies have been conducted on the $g h$ promoters of both Chinook salmon and rainbow trout $[22,23]$. Assembly of at least part of the transcriptional machinery was delineated to binding elements between positions -300 to -30. It was demonstrated that cyclic-AMP/PKA regulation of these promoters required nucleation and recruitment of both pituitary transcriptional activator-1 (Pit-1) and cAMP-response element (CRE) binding proteins (CREB) [22,23].

We note strong sequence identity of potential Pit-1, CREB, and retinoic acid receptor $(\mathrm{RAR}) /$ retinoid $\times$ receptor (RXR) binding elements that form the core of the proximal gh promoters of both Atlantic salmon (AS) and Chinook salmon (CS). An asymmetric CRE (TGACG) at -217/ -213 is flanked by at least four Pit-1 binding elements [see Additional file 1]. The more distal Pit-1 binding elements follow more strictly the core consensus sequence for Pit-1 $\left(\mathrm{W}_{3} \mathrm{CAT}\right)$ that is conserved in fish and mammals [24]. The potential RAR/RXR response element present at position 190/-171 could hypothetically permit interaction of chicken ovulbumin upstream promoter transcription factor (COUP-TF), or retinoic acid, estrogen or thyroid hormone receptors with an assembled Pit-1/CREB complex. Different variations of GTCT-rich blocks immediately upstream of each proximal promoter, as well as within the introns of each gene, may make regulatory or structural contributions to $g h$ transcription. For example, some of the CTCT blocks found in these regions could serve as binding elements for GAGA-binding factors [25].

However, differences do exist between the AS and CS gh1 and gh 2 promoters. For example, there is the potential for Pit- 1 to bind a reverse-oriented response element roughly $250 \mathrm{bp}$ further upstream in each $g h 2$ promoter that is not 


\section{Atlantic salmon}
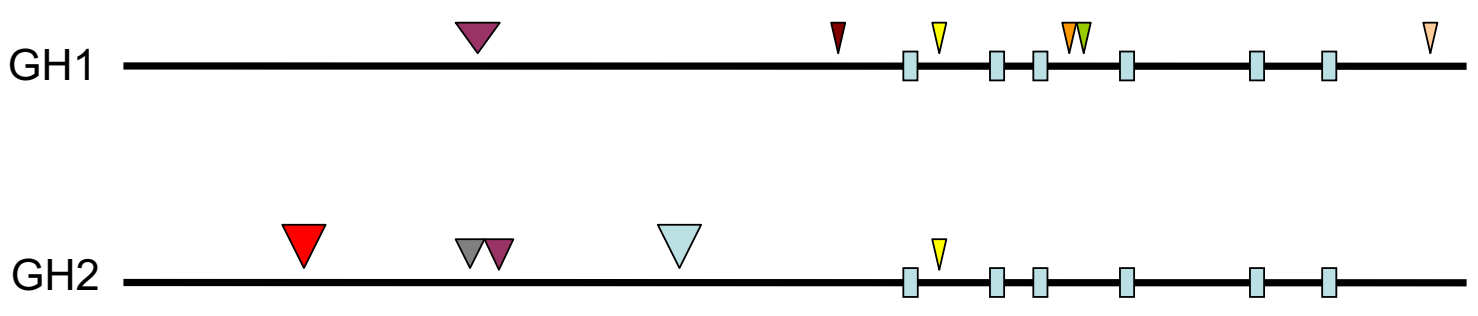

\section{Chinook salmon}

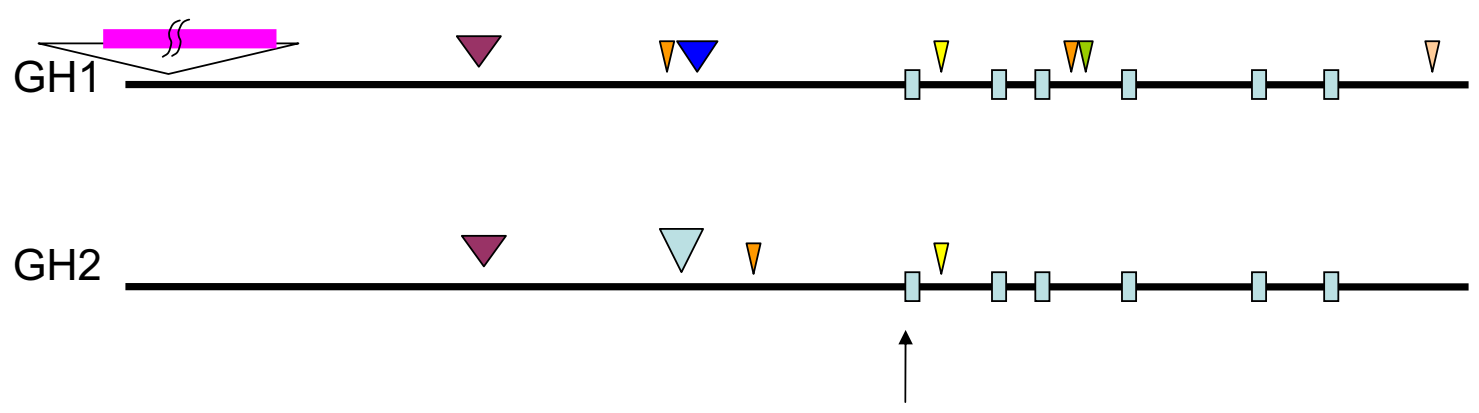

$\overline{1 \mathrm{~kb}}$

\section{Figure 3}

Transposon integration in Atlantic and Chinook salmon gh promoter regions. The insertion position of different transposon DNA sequences for each promoter are designated by coloured arrowheads and their corresponding family names. The arrow indicates the start of transcription, with approximately $7 \mathrm{~kb}$ shown for the promoter region, and $6 \mathrm{~kb}$ following the transcription start. Scale is approximate. Light blue boxes: exons I to 6. Purple arrowheads: DTSsa7 DNA transposon [Genbank:EF685960]; red arrowhead: DTSsa2 DNA transposon [Genbank:EF685955]; grey arrowhead: PTSsa I DNA transposon [Genbank:EF685966]; light blue arrowheads: Tss DNA transposon [Genbank:LI2207]; brown arrowheads: BHMS202 microsatellite [Genbank:AF256894]; dark blue arrowhead: SsaRT.3 LINE [unpublished]; orange arrowheads: Hpal SINE [Genbank:AY703447]; green arrowheads: Sssp220I microsatellite [Genbank:AY081807]; yellow arrowheads: SaSN2b SINE [unpublished]; cream arrowheads: C43 sequence repeat [unpublished]; purple rectangle: palindrome-containing Polinton-like insert ( $20 \mathrm{~kb}$ total).

present in the gh1 promoters. No palindromic CREs are found within $15 \mathrm{~kb}$ upstream of the gh genes, but one is present in intron 4 of the gh1 genes [see Additional file 1]. The different CRE, ERE and Pit-1 motif locations suggest that the gh1 and gh2 paralogues may have distinct regulatory modes and possibly act to function differently and/or at different times of salmonid development.

\section{GH expression and function}

The more traditional models that have regarded divergence among duplicate genes as a means for acquisition of new functions have met with recent challenges. For example, it has been demonstrated that mutation in many duplicated genes of rice is much slower than in 'singletons' and less drastic in terms of amino acid change [26]. Conservation of the apparent crucial functionality of the duplicates outweighs processes that lead to neofunctionalizaton. Indeed, it may be more common for gene nov- 
Growth Hormone 2

Chinook salmon

Atlantic salmon

\section{Growth Hormone 1}

Chinook salmon Atlantic salmon

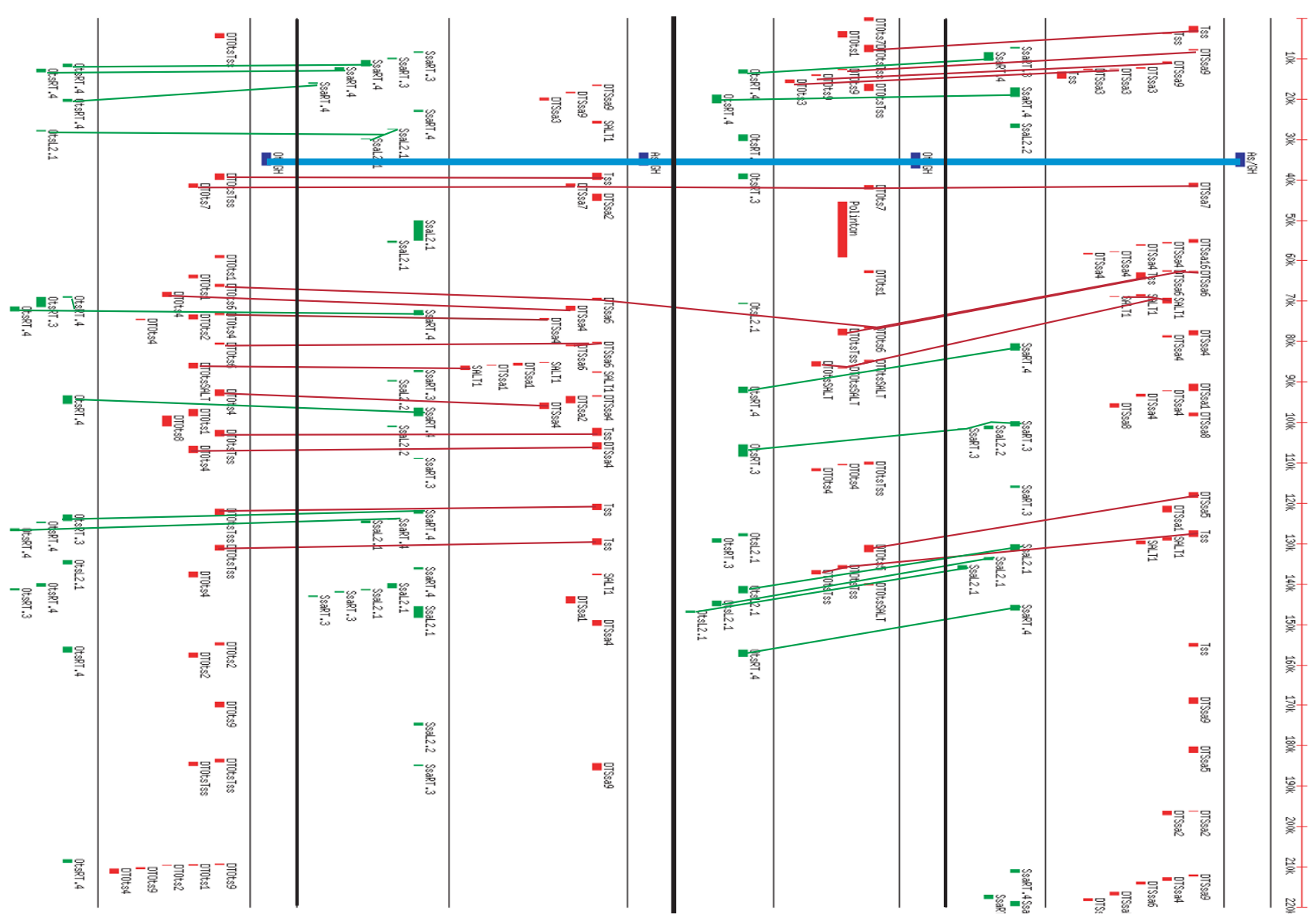

Figure 4

The distribution of DNA transposons and LINE sequence elements in gh I- and gh2-containing BACs. The growth hormone coding sequence (consisting of 6 exons) is aligned for the four BACs and connected by a light blue line. The conservation of the order of transposon families is indicated by lines, connecting corresponding sequences.

elty to arise from preserved duplicates that are subfunctionalized [27].

At the amino acid level, CS GH1 appears to be undergoing change much faster than any of the other GHs (Figure 2). In terms of retention of amino acid residue identity, the CS GH1 ORF has more changes than any of the other translated regions (nine differences overall compared to no more than three for any other gene). These changes do alter the polarity and charge of the proteins and therefore may subtly effect the kinetics and affinity of hormone binding to their receptor(s). We did find colocalized expression of both cDNAs in the CS gill and brain [see Additional files 2 and 3]. Interestingly, both GH receptors have been found in various regions of the brain and in the gill filaments of trout [10]. Furthermore, although the very limited amount of change between all four genes indicates that their functions are conserved, we do observe that GH1 and GH2 may potentially have different subfunctions. Our RT-PCR work demonstrates that some tissue expression segregation has occurred, whereby only gh2 appears to be expressed in muscle or eye [see Additional files 2 and 3].

To determine the function of locally expressed gh2 in the gill, it will be necessary to elucidate the specific cells GH synthesis is associated with and to determine whether local production is a component of known osmoregulatory processes or some other activity (see [7]). It is also possible that the gh2 expression observed in the gill is from leukocyte accumulation as leukocytes have been 
shown to produce both $g h 1$ and $g h 2$, with $g h 2$ the predominant transcript [28].

\section{Expression of genes linked with $\mathrm{GH}$}

Much less is known about the expression of the skeletal muscle sodium channel (scn), microtubule associated protein Tau (mapt), myosin alkali light chain $(\mathrm{mlc})$ and interferon alpha-1 (ifna1) genes in salmonids. To the best of our knowledge, for the other genes examined in this study, only the promoters of two ifn genes have been characterized in salmon. Two different AS type 1 ifn genes have been demonstrated to each possess two promoters that respond to different phases of infection or are tissue-specific in their transcriptional contexts [29].

The scn gene has been characterized in zebrafish [30] and fugu [31]. Some subfunctional partitioning of the four sets of duplicated zebrafish scn genes does exist, but there is still expression overlap across neuronal and muscle tissues [32]. The expression of the scn genes in the salmonids examined here also are not restricted to skeletal muscle, but their activities are found in a large number of different tissues [see Additional files 2 and 3].

There appears to be a number of ifn genes in both the Atlantic and Chinook salmon GH1 BACs analyzed (data not shown). At least one functional ifna1 gene and two to five different ifna genes (that might be pseudo or partial genes) exist at these loci for each species. It should be noted, however, that this region was difficult to sequence and assemble due to repetitive regions, therefore we cannot conclude unequivocally on the number of ifn genes present at these loci. A genomic BAC clone containing the ifna1 gene and seven other ifn genes has been characterized in Atlantic salmon (Robertsen, B: $7^{\text {th }}$ International Symposium on Fish Immunology. Abstract, 2007). However, to date, we find no other ifna genes present in our database other than ifna1 to enable us to conclude that expression of these other ifn genes does occur. Zebrafish also has two to three ifn genes in the 3 '-flanking region and the scn gene in the 5 '-flanking region of the gh gene on chromosome 3 [33].

\section{Genome rearrangements}

The gh2 genes have undergone some rearrangements in comparison to the gh1 paralogues (differences in promoter sequences, partly due to variable transposon integrations; deletions or lack of microsatellite insertions in intron 3; intronic microsatellite variations in intron 4). Part of this may be due to chromosome structure or positioning [8]. Also, some of the genomic variations observed between the gh1 and gh2 paralogues might be due to vestigial effects caused by a rearrangement that led to the rise of the Y-linked gh2 pseudogene [34]. Whether the additional transposon insertions found in the AS gh2 promoter can account for why we do see $g h$-Y in CS, but not in AS [35], will require characterization of other Pacific salmon $g h$ regions. It should be noted, as an example, that dramatic differences have also been found between the promoters of the salmon form of gonadotropin-releasing hormone in AS and sockeye salmon [36].

An indication of the changes that can occur when sequences of various transposon families integrate into the promoters of genes are shown in Figure 3. Insertions such as these may help to shape the dynamism of genomes by influencing modes of transcription through the introduction of new regulatory elements, by changing the distances between functional binding elements or by disabling them. It can be envisaged how alterations to transcription through these integration events could lead to changes in the function of their translated products over time. For example, note there are at least two different transposon sequences found within the AS gh2 proximal regulatory sequences that are not present in any of the other $g h$ promoters (Figure 3 ). These integrated sequences might impact the observed specific expression of $g h 2$ in AS compared to CS [see Additional files 2 and 3]. Also, the presence of a Tss transposon in the 5 ' sequence of each $g h 2$ and an HpaI short interspersed nuclear element (SINE) in intron 3 of each $g h 1$ is consistent with speciation events occurring after the gene or genome duplication events (Figures 3 and 4 ).

Vertebrate genomes contain large numbers of transposon sequences. The mouse and human genome contain approximately 3 million such sequences and zebrafish approximately 1.4 million. However, transposons occur in certain genome regions more frequently than in socalled transposon-free regions (which range from 5 to 66 $\mathrm{kb}$ in length), frequently associated with developmental genes $[37,38]$. This association with such genes suggests that transposons are deleterious to gene integrity possibly through increased mutagenesis or recombination, or by changing regulation. The presence of the Polinton sequence found upstream of the CS GH1 gene might therefore affect its genomic stability.

\section{Conclusion}

This is one of the first in a series of studies that are needed to document coding and non-coding changes that have occurred subsequent to a whole genome duplication. As GH has been the focus of biotechnological advances in aquaculture, it is important to investigate expression changes and genomic organizational changes in important economic traits such as growth. In this paper, we report on the impact of repeat elements and transposon integrations and show that in Oncorhynchus species the GH1 duplicate has undergone a higher rate of change. 
Genomes, particularly salmonid genomes, are dynamic. Part of the dynamism is the result of the purported whole genome duplication and the past integration of SINEs and LINEs into them. LINEs and SINEs introduce repetitive elements that may misalign causing unequal recombination to occur, and thereby introduce deletions and insertions among duplicated genomes. Coupled with this is the integration of a wide assortment of transposon sequences as described here and elsewhere [19]. Over time, these genomic rearrangements have led to the creation of pseudogenes, structural differences between duplicated genes and to differential regulation of paralogues.

In AS and CS, we show that transposon insertions have occurred differently in the promoters of $g h$, within and between each species. The organization of transposon families in the BACs containing gh1 and in the BACs containing gh2, is very similar within orthologous regions (Figure 4). However, much less conservation is apparent in comparisons between the gh1- and gh2-containing paralogous BACs for the two fish species (Figure 4). The appearance of repeated elements and differential rates of change in the gh1 and $g h 2$ regions is consistent with the hypothesis that a burst of transposition activity occurred during the speciation events which led to Atlantic and Pacific salmon [19]. Genome and gene duplication (30 to 100 MYA; $[39,40])$ has taken place much earlier than the speciation (14 to 23 MYA; $[9,41,42]$ ) and these data suggest that after gene duplication, gh1 in Oncorhynchus has evolved much faster than gh2, possibly because the genomic region for $g h 1$ has undergone more reorganization compared to the region containing gh2.

\section{Methods}

Atlantic salmon (AS) CHORI-214 [43] and Chinook salmon (CS) CHORI-217 [44] bacterial artificial chromosome (BAC) libraries were obtained from BACPAC Resources, Children's Hospital Oakland Research Institute (CHORI) [45]. AS BAC library filters were hybridized with an oligonucleotide probe (5'-TCCCAAACAAACAGCAACATACTCAACCGACCACCGCACT-3') designed from the AS gh1 EST [GenBank:X61938] that had been end-labeled with gamma- ${ }^{32}$ P-ATP (Amersham). The CS BAC library filters were screened using a $g h$ cDNA probe (GH2-8) generated from sockeye salmon genomic sequence by a PCR-based intron deletion methodology [35]. The CS GH2-8 probe was labelled with alpha-32P dCTP using the RediprimeII Random Prime Labelling system, and purified through a Probequant G-50 micro column (Amersham). Filter hybridizations were conducted as described by CHORI [45]. Probed BAC library filters were visualized using a Molecular Dynamics Storm PhosphorImaging system.

\section{BAC DNA confirmation}

Confirmation of AS $g h$-containing BACs was performed by comparisons of HindIII restriction digests of the isolated clones to in silico digests for each BAC. The entire Salmo salar genome BAC library has been digested by Hin$d \mathrm{III}$ and fragment profiles are available on the internet Contig Explorer version 3.4 (iCE 3.4) database [14]. Fingerprinting of the CS BACs was carried out by SnaPshot (ABI) labeling of restriction-digested fragments and samples were analyzed on an ABI 3130xl genetic analyzer. Data was processed utilizing Genemapper and fingerprinted contigs assembled as described by Luo et al [46].

BACs were assessed for whether they contained $g h 1$ or $g h 2$ using gene-specific polymerase chain reactions (PCRs). Primer sets were designed for AS gh1 intron 2 (5'-AAAACCAACGGCTCTTCAAC-3' and 5'-GGAGTCAGAGTTACAGAAGTCCAG-3'), intron 5 (5'-GATGACAATGACTCTCA GCAGC-3' and 5'-TGTATCTGGGAAACCGAACC-3'), as well as for $g h 2$ intron 3 (5'-ATCGTGAGCCCAATCGACAAGCAG-3' and 5'-GGGTACTCCCAGGATTCAATCAGG-3'). Primer sets were also designed for exon 3 (5'ACATGCAGCAGGATGCTAAG-3' and 5'-TTTCAGACCTTTATTGTCATCACC-3') and exon 5 (5'-GGTTCTGTGGAC ACTCAGTCC-3' and 5'-TCTTCGGAGGTGGCAAAG-3') of the sodium channel $(s c n)$ upstream of the gh genes. Detection of the $g h$ genes in the CS BACs was confirmed using the primer set 5'-AGCCTGGATGACAATGACTC-3' and 5'CTACAGAGTGCAGTTGGACT-3' which can distinguish all forms of $g h$ in the genome (GH1, GH2, and GH-P; [35]) and for the scn gene with 5'-TTCCGCCACTTCACCCTTG-3' and 5'-AGGGGCGTGTTGAACAGCTC-3'.

PCRs were performed using 200 ng of BAC DNA utilizing hot start PCRs: reaction mixtures without any enzyme were heated to $94^{\circ} \mathrm{C}$ for $3 \mathrm{~min}$, cooled to $80^{\circ} \mathrm{C}$ and then $0.625 \mathrm{U}$ of Taq DNA polymerase (Invitrogen) was added. Each PCR then underwent 35 cycles of the following parameters: $94^{\circ} \mathrm{C}$ for $20 \mathrm{sec}, 50^{\circ} \mathrm{C}$ for $30 \mathrm{sec}$ and $72^{\circ} \mathrm{C}$ for $45 \mathrm{sec}$. AS $g h 1$ was contained on BACs 11-I-04 and 73-D15 and gh2 in BAC 63-I-10. The CS gh1 was localized to BAC 108-O-24 and gh2 to BAC 206-E-17.

\section{BAC preparation and library construction}

BAC DNA was isolated by an alkaline lysis procedure using Nucleobond columns (Clontech) following the manufacturer's protocol. The isolated BAC DNA was nebulized and the DNA ends were made blunt by filling with T4 polymerase. The blunt-ended, repaired DNA was size fractioned by electrophoresis and the gel region corresponding to 1.6 to $4.0 \mathrm{~kb}$ was excised and gel purified (Qiagen). The fragments were blunt-end ligated into pUC19 plasmid cut with HincII (NEB) and transformed into electrocompetent DH5 $\alpha$ E. coli cells using a Bio-Rad Gene Pulser system. Library quality was evaluated and 
high redundancy plating was followed by large-scale colony picking (Genetix). Extracted recombinant plasmid templates were sequenced on an ABI 3730 DNA sequencer.

\section{BAC contig assembly}

Bases were called using PHRED $[47,48]$. High quality sequence reads were assembled using PHRAP [49] and then viewed and edited using Consed [50]. Some gaps in BAC assembly were filled by designing primers to the contiguous sequence ends, followed by amplification of the BAC region by PCR and subsequent cloning and sequencing of the fragments. Each BAC has been deposited in GenBank as follows: AS GH1 BAC 11-I-04 and BAC 73-D15 combined [GenBank:EU621898]; AS GH2 BAC 63-I10 [GenBank:EU621899]; CS GH1 BAC 108-O-24 [GenBank:EU621900] and CS GH2 BAC 206-E-17 [GenBank:EU621901].

Dotter [51] and PipMaker [52] were used to compare each BAC sequence to itself and to identify duplicated and repeated regions. Identification of other repeat elements was done with RepeatMasker [18] using repeat library 4.01 from Repbase [53], as well as a salmonid repeat database [19]. Gene location and direction on each BAC was determined using Digit Integrated Gene Identification Tools (DIGIT) [15].

\section{GH alignment, annotation, and comparison}

AS gh1 [GenBank:AY614010] and gh2 [GenBank:

M21573] gene sequences were used to align the gh1 and gh2 paralogues with BioEdit [54]. BioEdit was also used to annotate the two paralogues and calculate percent similarities between the two genes, their coding sequences, and their amino acid sequences. Searches and comparisons of regulatory elements for the two paralogues were also performed using BioEdit. Identification of transposon insertions was performed using Dotter plots, comparing $15 \mathrm{~kb}$ of each promoter region with salmon transposon sequences [19].

\section{Reverse transcription and cDNA amplification}

Total RNA was extracted in TRIzol reagent (Invitrogen) from flash-frozen, adult AS (Mowi stock, DFO, West Vancouver) and CS (Chehalis River Hatchery) kidney, muscle, skin, gut, gill, spleen, brain, heart, testis, liver, eye and pyloric caecum tissues. The extracted total RNAs were cleaned using MEGAclear (Ambion) and then quantified and quality-checked by spectrophotometer and agarose gel, respectively.

The cDNAs were synthesized in $25-\mu \mathrm{L}$ reactions from 1.0 $\mu \mathrm{g}$ total RNA using oligo(dT) $)_{15}$ (Promega) and Supercript II RNase $\mathrm{H}^{-}$reverse transcriptase according to the manufacturer's instructions (Invitrogen). The reactions were incubated at $37^{\circ} \mathrm{C}$ for $90 \mathrm{~min}$ and the transcriptase heatinactivated at $70^{\circ} \mathrm{C}$ for $30 \mathrm{~min}$. Approximately $200 \mathrm{ng}$ of cDNA was used in each $25-\mu \mathrm{L}$ PCR reaction containing 1.25 U Taq polymerase, $1 \times$ Taq buffer, $1.25 \mathrm{mM} \mathrm{MgCl}_{2}$, $10 \mathrm{mM}$ dNTPs (Invitrogen) and $15 \mathrm{pmol}$ of each genespecific 5' and 3' primer [see Additional file 4]. Each PCR was carried out under the following cycling parameters: $94^{\circ} \mathrm{C}$ for $2 \mathrm{~min}$, then 35 cycles of $94^{\circ} \mathrm{C}$ for $30 \mathrm{sec}, 55^{\circ} \mathrm{C}$ for $30 \mathrm{sec}$, and $72^{\circ} \mathrm{C}$ for 1 min using a Perkin Elmer 9600 . The AS scn 1 and scn 2 PCRs were similarly amplified but the anneal temperature used was $57.5^{\circ} \mathrm{C}$. The integrity of each cDNA used was confirmed by control PCRs using ubiquitin primers.

The PCR products were separated by electrophoresis on 1.25 to $1.30 \%$ agarose gels and photographs were stored using an UVP GelDoc-It documentation system (UVP). Representative products were isolated and cloned into pCR2.1-TOPO vector (Invitrogen) and sequenced to confirm gene identities.

\section{Authors' contributions}

KRVS: performed tissue expression RT-PCRs, gh promoter analysis and drafted the manuscript. RY: performed BAC sequence data analysis and annotations. JdB: performed transposable element analysis. KPL: designed probes and performed hybridizations to identify AS gh-containing BACs. BG: designed probes and performed hybridizations to identify CS $g h$-containing BACs. CAS: performed initial sequencing and analysis of Atlantic salmon $g h$-containing BACs for Biology Honours thesis MRB-S: and AR: performed clone preparation and sequencing. WSD, RHD and BFK: obtained funding, and contributed to experimental design, analysis and writing of the manuscript. All authors read and approved the final manuscript.

\section{Additional material}

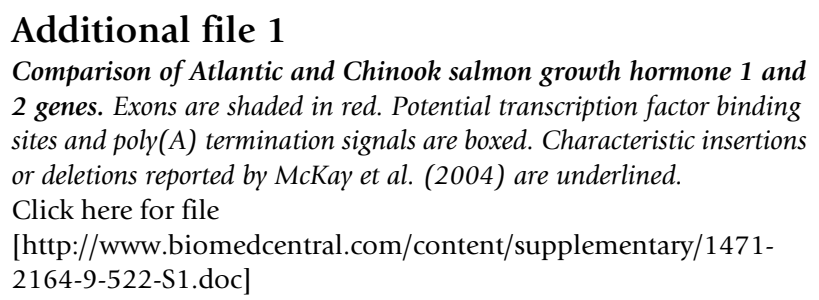




\begin{abstract}
Additional file 2
Reverse transcriptase PCR validation and cDNA expression profiles in twelve different tissues. Reverse transcriptase PCR validation and cDNA expression profiles in twelve different tissues: 1: kidney, 2: muscle, 3: skin, 4: gut, 5: gill, 6: spleen, 7: brain, 8: heart, 9: testis, 10: liver, 11: eye and 12: pyloric caecum. The integrity of each cDNA used was confirmed by control PCRs using ubiquitin primer set. For each gene-specific PCR experiment, a negative control with no template (NC) was included. Abbreviations for gene names are as follows: GH: growth hormone; Scn: skeletal muscle sodium channel alpha subunit; Mlc: myosin alkali light chain; IFN: interferon. The strongest marker band indicates a fragment length of $500 \mathrm{bp}$.

Click here for file

[http://www.biomedcentral.com/content/supplementary/14712164-9-522-S2.ppt]
\end{abstract}

\section{Additional file 3}

Reverse transcriptase PCR validation and cDNA expression profiles in twelve different tissues. Reverse transcriptase PCR validation and cDNA expression profiles in twelve different tissues: 1: kidney, 2: muscle, 3: skin, 4: gut, 5: gill, 6: spleen, 7: brain, 8: heart, 9: testis, 10: liver, 11: eye and 12: pyloric caecum. The integrity of each cDNA used was confirmed by control PCRs using ubiquitin primer set. For each gene-specific PCR experiment, a negative control with no template (NC) was included. Abbreviations for gene names are as follows: GH: growth hormone; Scn: skeletal muscle sodium channel alpha subunit; Mlc: myosin alkali light chain; IFN: interferon. The strongest marker band indicates a fragment length of $500 \mathrm{bp}$.

Click here for file

[http://www.biomedcentral.com/content/supplementary/1471-

2164-9-522-S3.ppt]

\section{Additional file 4}

Gene-specific primer oligonucleotide sequences used in tissue expression studies. A list of primer identification and primer sequences for each amplified gene of interest are provided.

Click here for file

[http://www.biomedcentral.com/content/supplementary/14712164-9-522-S4.xls]

\section{Acknowledgements}

This research was supported by Genome Canada, Genome BC and the Province of $\mathrm{BC}$, and additionally by the Natural Sciences and Engineering Research Council of Canada (BFK, WSD), and the Canadian Federal Government Genomics Research and Development Initiative (RHD). We are indebted to Carlo Biagi for collection of the AS and CS tissues.

\section{References}

I. Thorgaard GH, Bailey GS, Williams D, Buhler DR, Kaattari SL, Ristow SS, Hansen JD, Winton JR, Bartholomew JL, Nagler JJ, Walsh PJ, Vijayan MM, Devlin RH, Hardy RW, Overturf KE, Young WP, Robison $B D$, Rexroad C, Palti Y: Status and opportunities for genomics research with rainbow trout. Comp Biochem Physiol B Biochem Mol Biol 2002, 133:609-646.

2. Groot C, Margolis L: Pacific Salmon Life Histories Vancouver, Canada: UBC Press; 1991.

3. Groot C, Margolis L, Clarke WC: Physiological Ecology of Pacific Salmon Vancouver, Canada: UBC Press; 1995.

4. Thompson BJL, Shang CA, Waters MJ: Identification of genes induced by growth hormone in rat liver using cDNA arrays. Endocrinology 2000, 141:4321-4324.
5. Bjornsson BTh: The biology of salmon growth hormone: from daylight to dominance. Fish Physiol Biochem 1997, 17:9-24.

6. Kraak G van der, Rosenblum PM, Peter RE: Growth hormonedependent potentiation of gonadotropin-stimulated steroid production by ovarian follicles of the goldfish. Gen Comp Endocr 1990, 79:233-239.

7. Sakamoto T, Hirano T: Expression of insulin-like growth factor I gene in osmoregulatory organs during seawater adaptation of the salmonid fish: Possible mode of osmoregulatory action of growth hormone. Proc Natl Acad Sci USA 1993, 90:1912-1916.

8. McKay SJ, Trautner J, Smith MJ, Koop BF, Devlin RH: Evolution of duplicated growth hormone genes in autotetraploid salmonid fishes. Genome 2004, 47:714-723.

9. Devlin RH: Sequence of sockeye salmon type I and 2 growth hormone genes and the relationship of rainbow trout with Atlantic and Pacific salmon. Can J Fish Aquat Sci 1993, 50:1738-1748.

10. Very NM, Kittilson JD, Norbeck LA, Sheridan MA: Isolation, characterization, and distribution of two cDNAs encoding for growth hormone receptor in rainbow trout (Oncorhynchus mykiss). Comp Biochem Physiol B 2005, 140:615-628.

II. Baudet ML, Martin B, Hassanali Z, Parker E, Sanders EJ, Harvey S: Expression, translation and localization of a novel, small growth hormone variant. Endocrinology 2007, I 48:103-I15.

12. Mori T, Devlin RH: Transgene and host $\mathbf{G H}$ gene expression in pituitary and nonpituitary tissues of normal and GH transgenic salmon. Mol Cell Endocrinol 1999, 149:129-139.

13. Yada T, Azuma T: Hypophysectomy depresses immune functions in rainbow trout. Comp Biochem Physiol C 2002, 13 1:93-100.

14. iCE - internet Contig Explorer [http://www.bcgsc.ca/platform/ bioinfo/software/ice]

15. DIGIT - Digit Integrated Gene Identification Tools [http:// digit.gsc.riken.jp/cgi-bin/index.cgi]

16. Altschul SF, Madden TL, Schäffer AA, Zhang J, Zhang Z, Miller W, Lipman DJ: Gapped BLAST and PSI-BLAST: a new generation of protein database search programs. Nucleic Acids Res 1997, 25:3389-3402.

17. Frazer KA, Pachter L, Poliakov A, Rubin EM, Dubchak I: VISTA: computational tools for comparative genomics. Nucleic Acids Res 2004, 32:W273-279.

18. RepeatMasker Open - 3.0 [http://www.repeatmasker.org]

19. de Boer JG, Yazawa R, Davidson WS, Koop BF: Bursts and horizontal evolution of DNA transposons in the speciation of pseudotetraploid salmonids. BMC Genomics 2007, 8:422-43I.

20. Lukacs MF, Harstad H, Grimholt U, Beetz-Sargent M, Cooper GA, Reid L, Bakke HG, Phillips RB, Miller KM, Davidson WS, Koop BF: Genomic organization of duplicated major histocompatibility complex class I regions in Atlantic salmon (Salmo salar). BMC Genomics 2007, 8:25।-266.

21. Kapitonov VV, Jurka J: Self-synthesizing DNA transposons in eukaryotes. Proc Natl Acad Sci USA 2006, I 03:4540-4545.

22. Argenton F, Vianello S, Bernardini S, Lopreiato R, Colombo L, Bortolussi $M$ : Trout $\mathbf{G H}$ promoter analysis reveals a modular pattern of regulation consistent with the diversification of $\mathbf{G H}$ gene control and function in vertebrates. Mol Cell Endocrinol 2002, 189: II-23.

23. Wong AOL, Le Drean Y, Liu D, Hu ZZ, Du SJ, Hew CL: Induction of Chinook salmon growth hormone promoter activity by the adenosine $3^{\prime}, 5^{\prime}$-monophosphate (cAMP)-dependent pathway involves two cAMP-response elements with the CGTCA motif and the pituitary-specific transcription factor Pit-I. Endocrinology 1996, 137:1775-1784.

24. Andersen B, Rosenfeld MG: Pit-I determines cell types during development of the anterior pituitary gland. J Biol Chem 1994, 269:29335-29338.

25. Lehmann M: Anything else but GAGA: a nonhistone protein complex reshapes chromatin structure. Trends Genet 2004, 20:15-22.

26. Chapman BA, Bowers JE, Feltus FA, Paterson AH: Buffering of crucial functions by paleologous duplicated genes may contribute cyclicality to angiosperm genome duplication. Proc Natl Acad Sci USA 2006, 1 03:2730-2735.

27. Lynch $M$, Force $A$ : The probability of duplicate gene preservation by subfunctionalization. Genetics 2000, 154:459-473. 
28. Yada T, Muto K, Azuma T, Hyodo S, Schreck CB: Cortisol stimulates growth hormone gene expression in rainbow trout leucocytes in vitro. Gen Comp Endocrinol 2005, 142:248-255.

29. Bergan V, Steinsvik S, Xu H, Kileng O, Robertsen B: Promoters of type I interferon genes from Atlantic salmon contain two main regulatory regions. FEBS Journal 2006, 273:3893-3906.

30. Novak AE, Jost MC, Lu Y, Taylor AD, Zakon HH, Ribera AB: Gene duplications and evolution of vertebrate voltage-gated sodium channels. J Mol Evol 2006, 63:208-22I.

31. Fugu Genomics Project [http://fugu.biology.qmul.ac.uk]

32. Novak AE, Taylor AD, Pineda RH, Lasda EL, Wright MA, Ribera AB: Embryonic and larval expression of zebrafish voltage-gated sodium channel alpha-subunit genes. Dev Dyn 2006, 235: 1962-1973.

33. Zebrafish Genome Resources [http://www.ncbi.nlm.nih.gov/ genome/guide/zebrafish]

34. Du SJ, Devlin RH, Hew CL: Genomic structure of growth hormone genes in Chinook salmon (Oncorhynchus tshawytscha): presence of two functional genes, $\mathrm{GH}-\mathrm{I}$ and $\mathrm{GH}-\mathrm{II}$, and a male-specific pseudogene, GH-psi. DNA Cell Biol 1993, | 2:739-75|.

35. Devlin RH, Biagi CA, Smailus DE: Genetic mapping of Y-chromosomal DNA markers in Pacific salmon. Genetica 200I, I I I:43-58

36. Coe IR, von Schalburg KR, Sherwood NM: Characterization of the Pacific salmon gonadotropin-releasing hormone gene, copy number and transcriptional start site. Mol Cell Endocrinol 1995, I I5: I13-122.

37. Simons C, Pheasant M, Makunin IV, Mattick JS: Transposon-free regions in mammalian genomes. Genome Res 2006, I6: I64-I 72.

38. Simons C, Makunin IV, Pheasant M, Mattick JS: Maintenance of transposon-free regions throughout vertebrate evolution. BMC Genomics 2007, 8:470-479.

39. Allendorf FW, Thorgaard GH: Tetraploidy and the evolution of salmonid fishes. In Evolutionary Genetics of Fishes Edited by: Turner BJ. New York: Plenum Press; 1984: I-53.

40. Hoegg S, Brinkmann H, Taylor JS, Meyer A: Phylogenetic timing of the fish-specific genome duplication correlates with the diversification of teleost fish. J Mol Evol 2004, 59:190-203.

4I. McKay SJ, Devlin RH, Smith MJ: Phylogeny of Pacific salmon and trout based on growth hormone type-2 and mitochondrial NADH dehydrogenase subunit 3 DNA sequences. Can J Fish Aquatic Sci 1996, 53: I 165-1 I76.

42. Oleinik AG: On the mutation rates of the mitochondrial and nuclear genomes of salmonid fishes. Russian Journal of Marine Biology 2000, 26:432-438.

43. CHORI - 2/4 - Children's Hospital Oakland Research Institute - $2 / 4$ [http://bacpac.chori.org/library.php?id=102]

44. CHORI - 217-Children's Hospital Oakland Research Institute -217 [http://bacpac.chori.org/library.php?.id=223]

45. CHORI - Children's Hospital Oakland Research Institute [http://bacpac.chori.org/highdensity.htm]

46. Luo M-C, Thomas C, You FM, Hsiao J, Ouyang S, Buell CR, Malandro M, McGuire PE, Anderson OD, Dvorak J: High-throughput fingerprinting of bacterial artificial chromosomes using the SnaP. shot labeling kit and sizing of restriction fragments by capillary electrophoresis. Genomics 2003, 82:378-389.

47. Ewing B, Green P: Base-calling of automated sequencer traces using PHRED. II. Error probabilities. Genome Res 1998, 8:186-194

48. Ewing B, Hillier L, Wendl MC, Green P: Base-calling of automated sequencer traces using PHRED. I. Accuracy assessment. Genome Res 1998, 8:175-185.

49. PHRAP [http://bozeman.mbt.washington.edu]

50. Gordon D, Abajan C, Green P: Consed: a graphical tool for sequence finishing. Genome Res 1998, 8:195-202.

5I. Sonnhammer EL, Durbin R: A dot-matrix program with dynamic threshold control suited for genomic DNA and protein sequence analysis. Gene 1995, 167:GCI-10.

52. Schwartz S, Zhang Z, Frazer KA, Smit A, Riemer C, Bouck J, Gibbs R, Hardison R, Miller W: PipMaker - a web server for aligning two genomic DNA sequences. Genome Res 2000, 10:577-586.

53. Jurka J: Repbase update: a database and an electronic journal of repetitive elements. Trends Genet 2000, 16:418-420.

54. BioEdit [http://www.mbio.ncsu.edu/BioEdit/bioedit.html]

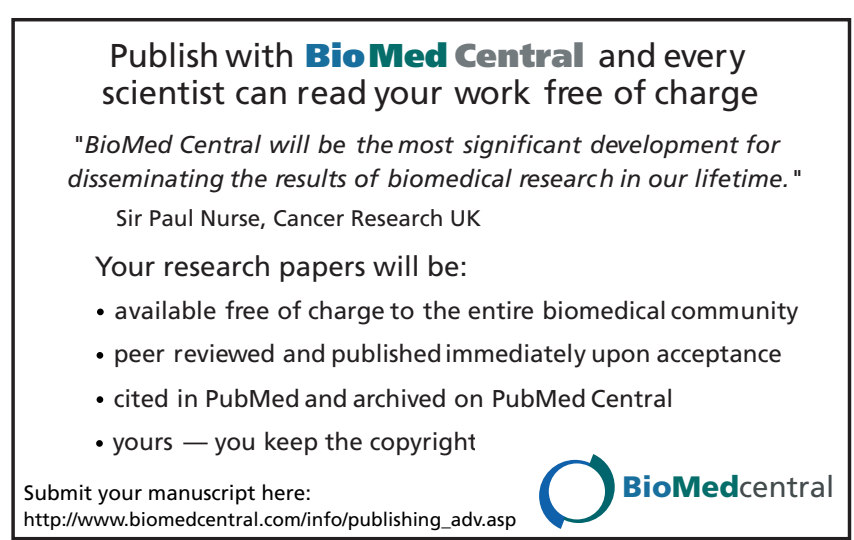

